\title{
Dietary consumptions pattern and risk of oral cancer: A Malaysian scenario
}

Type: Meeting Abstract.

\section{Content:}

Introduction: The risk of cancer has been extensively investigated with the intake of single food items but food consumption in an individual is usually complex, made up by combinations of different food items. This study aims to identify dietary patterns of Malaysians, and establishing relationship between dietary pattern and oral cancer risk. Methods A hospital-based, case-control study was conducted on 300 Malaysians who seek treatment at participating hospitals between January 2003 and December 2010. The subjects consisted of 150 cases and 150 controls that were matched for gender, age (5 years) and ethnicity. Dietary intake was measured using Food Frequency Questionnaire (FFQ). Factor Analysis (FA) was performed to identify dietary consumptions pattern based on the intake of nine major food groups. Logistic Regression was done to compute the odds ratio $(\mathrm{OR})$ for the components retained by FA. Results: FA had identified four patterns that accounted for $69.4 \%$ of total variability within the sample. The first pattern labeled as 'modern' was loaded with processed foods and snacks intakes whereas the second pattern 'prudent' was characterized by the intakes of fruits and vegetables. The third pattern 'traditional' consisted of beverages and starches intakes while the fourth pattern 'combination' was loaded with intakes of dairy foods, fermented foods and meat/byproducts. A significant reduced risk was found for 'prudent' (aOR $0.42,95 \% \mathrm{Cl}: 0.21-0.83, \mathrm{p}<0.05)$, whereas a significant increased risk was found for both 'traditional' (aOR 2.06, 95\% Cl: 1.05-4.02) and 'combination' (aOR 3.45, 95\% Cl: 1.75-6.77, $\mathrm{p}<0.05$ ) pattern. Discussion: These findings suggest that intake of 'prudent' pattern may confer protection against oral cancer risk of up to $58 \%$ whereas the highest consumption of both 'traditional' and 'combination' pattern may induce twice and thrice the risk of oral cancer. FA was also found to be a useful tool to identify dietary pattern and its relationship with oral cancer.

\begin{tabular}{|c|l|}
\hline Author & $\begin{array}{l}\text { Helen-Ng, L. C. ; Ishak, I. A. ; Ghani, W. M. N. ; Marhazlinda, J. ; } \\
\text { Norlida, A. ; Zain, R. B. }\end{array}$ \\
\hline Source & Oral Oncology \\
\hline ISSN & $1368-8375$ \\
\hline DOI & $10.1016 /$ j.oraloncology.2011.06.341 \\
\hline Volume & 47 \\
\hline Page & S105-S105 \\
\hline Year & 2011 \\
\hline
\end{tabular}


Keyword:

Oral squamous cell carcinoma, OSCC, lichenoid lesions, lichen planus, oral cancer, oral tumours, pemphigus, traumatic eosinophilic granuloma, aphthous ulcers, oral mucosal lesions, betel chewers mucosa, betel quid related lesions, betel quid, areca quid, tobacco quid, oral cancer screening, training and calibration, early detection, oral cancer awareness, biobanking, tissue bank, databank, oral cancer, tissue bank, research credibility, research ethics.

Please Cite As:

HELEN-NG, L. C., ISHAK, I. A., GHANI, W. M. N., MARHAZLINDA, J., NORLIDA, A. \& ZAIN, R. B. 2011. Dietary consumptions pattern and risk of oral cancer: $A$ Malaysian scenario. Oral Oncology, 47, S105-S105.

URL:

- http://apps.webofknowledge.com/full record.do?product=WOS\&search mode=M arkedList\&qid=20\&SID=4DCLI422CFMEHc1pFfd\&page $=1 \& d o C=1 \&$ colName $=W$ $\underline{\mathrm{OS}}$

- $\quad$ http://www.sciencedirect.com/science/article/pii/S1368837511005471 